\title{
The Application of the Countertenor Technique in Chinese Pop Songs Training
}

\author{
Yanxi Chang \\ Mahasarakham University, Kantharawichai, Thailand \\ Email: 54165796@qq.com
}

How to cite this paper: Chang, Y. X. (2018). The Application of the Countertenor Technique in Chinese Pop Songs Training. Art and Design Review, 6, 133-140. https://doi.org/10.4236/adr.2018.63013

Received: May 21, 2018

Accepted: August 5, 2018

Published: August 8, 2018

Copyright $\odot 2018$ by author and Scientific Research Publishing Inc. This work is licensed under the Creative Commons Attribution International License (CC BY 4.0).

http://creativecommons.org/licenses/by/4.0/

\begin{abstract}
In recent years, more and more countertenors have been recognized, accepted and loved by the public in China. There have also been a number of excellent singers in pop music with countertenor skills. Therefore, we discussed the four steps about training countertenor techniques, and it is of practical significance to study the vocal music teaching of this relatively special technique, and it can enrich the singing skills and style of pop singers. The combination of technical theory and aesthetic appreciation is an effective method to effectively train countertenor in popular songs, and it can help students to raise their confidence in singing countertenor and improve the performance of students.
\end{abstract}

\section{Keywords}

Countertenor, Pop Songs Performance, Vocal Training, Pitch Pipe, Weak Voice Training

\section{Introduction}

The countertenor, like tenor and soprano, is also a kind of sound in the field of western classical vocal music. The countertenor is usually defined as the highest tenor in the highest part of the voice. In other words, this kind of sound has already exceeded the tenor's range in the general sense, and to some extent it coincides with the range of alto, mezzo-soprano and even soprano. Therefore, the countertenor is also divided into the countertenor similar to the alto, the falsetto tenor similar to mezzo-soprano, and the falsetto tenor similar to the soprano.

The countertenor is an adult male who, on the basis of natural sound, relies on freedom to move to a high position of the larynx, and to sing the height of the female voice in a resonant head. The sound quality always maintains the 
characteristics of the male voice, and it is not as weak as the female in the high pitch area, but it is clear and transparent, and the range is very wide. This kind of special vocal singing training is not like a eunuch singers to borrow physiological procedure, there is a difference in tone between them, but there has been a habit of writing for this role since the middle age.

It was early in the year 367 A.D. that the Catholic Church stripped the people of their right to sing at religious gatherings, and to be arranged by the trained choir. In addition, "women must remain silent in the church", so the singing in a boy's voice is in the choir, but they have to face the natural selection of the mutation. So falsetto singers replaced the boys in some churches, the falsetto singers from Spain have the secret to sing the sopranos, and then music scholars find out that they are Castrato. The castrated singers occupied the stage of the opera in the sixteenth and seventeenth centuries with their superb vocal technique; this inhumane method was destined to be the ashes of history as time goes by. In the latter part of the twentieth century, many of the songs sung by the eunuchs were performed by the female or falsetto tenor. As a result of the study of ancient music, the vocal technique of castrating was retained. Through special training, the countertenor returned to the stage.

\section{The Countertenor Performance in Pop Songs in China}

Countertenors are not uncommon. Due to the increasing diversity of media means, more and more countertenors are beginning to be recognized, accepted and loved by the public. Such as German countertenor Andreas Scholl, American countertenor David Daniels and French countertenor Philippe Jaroussky are currently active in the international famous falsetto tenor of classical music. Countertenors play an important role not only in classical music, but also in popular music. For example, the Russian prince of dolphin vocal sound, Bumac Vitas, and the British classical crossover singer, the King's Singers, are renowned in pop music world today. In China there are also a lot of very good countertenors, for example, Xiao Ma and Li Yugang, who are the representatives of our country's countertenors. Xiao Ma won the second place in China's sixth international vocal music competition in 2014, and Li Yugang is a well-known singer who is familiar with female vocals. Therefore, the vocal music teaching of countertenor is of practical significance.

Although traditionally countertenor has ever chosen more confined to the baroque and classical periods of vocal music composers, such as Purcell, Monteverdi, Handel, Bach, Scarlatti, Mozart, etc. But in recent years, the countertenor has become increasingly recognized and accepted by audiences on the international opera stage. Therefore, many different styles of vocal music works in other periods can be used as the music of the countertenor study and singing, for example, German art songs, French art songs, Chinese art songs, etc. The Chinese art songs, "on the water side", "I live upstream by the Yangtze river", "the apricot blossom and shadow everywhere", "red bean words", and so on, are very 
suitable for the countertenor to sing. Xiao Ma is Chinese first high countertenor singer. Through years of learning, he can learn a lot of Chinese and foreign operas, Chinese art songs and musical works and he also has a unique singing style, in the interpretation of classical and modern music elements combined with the vocal music works.

Nan Dan is a man who plays the woman characters, and the vocal technique of Nan Dan in Pecking opera is similar with the vocal technique of countertenor, but the style is different. Li Yugang is one of the most famous Nan Dan actors in China, he can use the Nandan vocal technique in his Chinese pop song "Drunken beauty", and he also can use the countertenor technique in the Russian art song "A night outside Moscow" with pop music style, because the vocal technique is similar, just different style.

Now some Chinese singers can make dolphin vocal sound like Vitas and apply this technique to songs. Actually the male singer's dolphin vocal sound technique is the countertenor, which is only different in style and pitch. The Chinese singer Wang Yuexin can sing many of Vitas' works, and use the countertenor technique in his popular song "light".

\section{Four Steps to Train the Countertenor of Pop Songs}

When we use in popular singing countertenor technology, not only can we enrich popular singing style and color, also we can make popular singing training and voice method more scientific, science of popular singing method can extend the voice range, even to join countertenor style to the pop music voice works. We require students to study by learning a lot of famous countertenors singing audio and video data, to make the students gradually to produce a deep impression about the pure head sound of the countertenor singing. Then I have four steps in the class to train the countertenor in pop song, with the weak voice training.

\subsection{Establishing a Body Instrument}

To train countertenor, we need five movements to build a body instrument. When we apply countertenor technology in popular songs, we need to build the same instrument, and it is the resonance body cavity and the state of singing. The state of the singing, the intensity of the vocal cords, and the control of the breath, are proportional, the ratio is " $7: 2: 1$ ".

When we breathe in control, we are proactive attitude, with one hundred percent of the state. so our efforts in the proportion of the vocal cords must reach the breath twice, and the condition of voice also must reach the breath the strength of the 7 times, if the voice state haven't reach the strength, so the vocal cords and breath will be made according to the proportion of the " $7: 2: 1$ " cut. So the intensity of our vocal cords must be twice the breath, and the sound state must be seven times the intensity of the breath. If the vocal state has not yet reached this level, the intensity of the vocal cords and breath should be reduced, 
by the ratio of "7:2:1".

First of all, the throat should be pulled up and down. The epiglottis should be curled up backwards the pharyngeal wall to establish the Pitch pipe (for example: Figure 1). There is a gap between throat bone and thyroid bone, we should pull the throat bone to the occipital bone in your awareness and pull the thyroid bone to the tailbone. When the students sing, the sound must hang in the nasopharyngeal cavity and uvula first, and yawn to lift up your soft palate and relax your jaw. Second, the throat should be opened back and forth, especially bottom of throat. The student should feel to sign in front of throat, and feel to emesis at the back of throat. Third, the thoracic cavity should be expanded, and the student should feel a whirl pool in your thoracic cavity. Fourth, the ribs should be expanded, and the students should feel the epigastrium to plump up. Fifth, the students should feel the waist like a swim ring, and the hypogastrium should be shrunk a little. The hipbone is the axis, the student should feel the strength to lift upwards and turn backwards around the axis.

When the students sing, the student should feel someone pushing them, and their feet take root on the floor firmly, their body must lean forward, but resist the power behind. The sound is on the heel, the thoracic cavity, ribs, the nasopharyngeal cavity and uvula, the students should feel the thoracic cavity on their heels (Stein, 1959). To set up the vocal music instrument, we should imagine the four mouths of the singing state: the nasopharyngeal cavity, the posterior pharyngeal wall, the chest, and the back of the waist, which are the four positions of the singing state. The sound falls on the back of the waist, and the front ribs are propped up, and we actively apply the diaphragm, like a sigh. The back of the neck and the back of the waist are two weak spots, and the correct method is needed to put them on the right position and correctly focus on the strength.

\subsection{Weak Voice Training}

To sing countertenor, we need to use weak voice training. With long-term training,

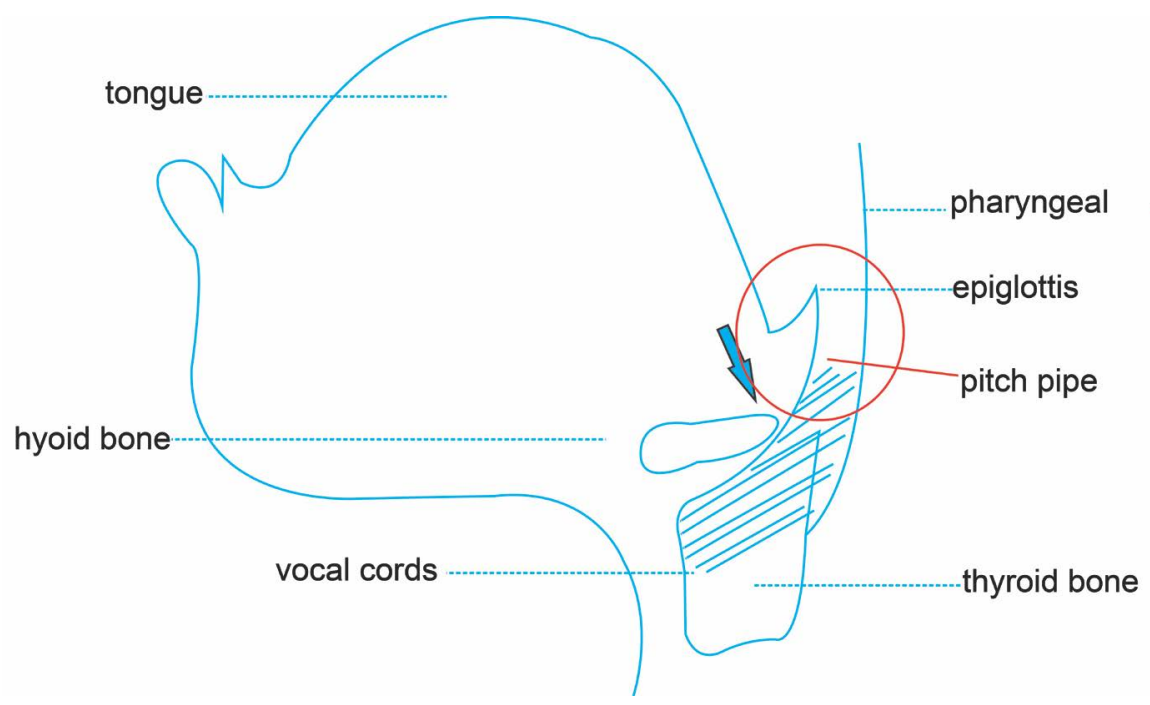

Figure 1. The pitch pipe location picture. 
this weak sound function will be strengthened, and the pitch pipe will become more and more powerful, and the volume will gradually enlarge. In classical songs, the sound needs to be released, while in the pop singing process, the weak vocal performance is the main, and then the microphone should be used to expand.

The students should not inhale fully, otherwise breath cannot circulate, and button their lips and hum in voice crying: " $m$ ", then yawn and phonate "mo", which is in the pitch pipe, not in the mouth. When they sing "mo" in arpeggios exercise or pop song melody, the students should adjust their body instrument.

First, Breath adjustment.

Because of the voice range of the countertenor is wide, countertenor breath adjustment is particularly important, this kind of application of breath in pop songs, is not only beneficial to sing the popular style of countertenor, more conducive to the treble breath control and the stability of the breath of popular songs in the bass.

The breathing in singing is not the same as the breathing in daily life. In daily life, people speak to communicate thoughts and feelings, with small volume and shallow breath because the close average distance. And we speak for a long time, and our voice is tired and hoarse, and this kind of breathing is not sufficient for singing.

Therefore, the breath of singing as an artistic means, has its own unique set of methods, it is a technical problem, and need to be trained. While performing the five movements of the body instrument, while we sing the lower pitch like sighing, sing the middle pitch like talking, and sing the higher pitch like shouting. When we sing the lowest voice, the sound position will land on the tailbone. As the pitch goes up, the position of the sound is pulled down from the tailbone, and when it reaches the highest pitch, the sound position circulates from the land to the sky (Burkholder, 2006).

I take gong as an example, when we strike a gong, we can hear the same pitch no matter how we use great strength. If we want to get higher pitch, we must change a smaller gong. If we want to get higher pitch with any instrument, we must adjust the instrument such as piano, violin and so on. Therefore, when singing a high pitch, you can't use the fully breath to go up and you should lose half of the breath. It should be in accordance with the ratio of " $7: 2: 1$ ", the state is greater than the vocal cords, and the vocal cords is more than the breath.

Second, the students should adjust their pitch pipe. When they are practicing their body instruments from pitch $\mathrm{C} 1$ to $\mathrm{C} 5$, the students should sing "mo" with the "A pitch pipe", and sing "mo" with the "O pitch pipe" from pitch bA1 to E2, with the "U pitch pipe" from F2 to G2, with the small "O pitch pipe" at bA2, and with the "A pitch pipe" from A2 to C4. The" A pitch pipe" is narrower than the "small O pitch pipe", the "small O pitch pipe" is narrower than "O pitch pipe", and the "O pitch pipe" is narrower than the " $U$ pitch pipe". When we sing pop songs, we train the students to adjust the pitch pipe narrower and narrower, just 
with "A pitch pipe". We apply the finest "A pitch pipe", over the high pitch C3, and we also can apply the "O pitch pipe", the "small O pitch pipe" and the "U pitch pipe" to add the countertenor style in pop songs.

Third, the students should adjust their voice cords. The teacher should teach the students to adjust their voice cords thinner and thinner when they sing from lower pitch to higher pitch. And we should adjust the vocal cords muscle and the cricothyreoideus muscle. The vocal cords muscle is always in a state of constant contraction, from a lower pitch to a higher pitch. The higher the pitch is, the stronger the contraction is, and the shorter the vocal cords are, and the cricothyreoideus muscle should always be in a passive state.

When we sing the classical song, we should teach the students adjust the voice cords from the wholly-body vibration to the local vibration from the pitch $\mathrm{C} 1$ to the pitch E2, from the local vibration to the edge vibration from the pitch E2 to the left pitch, for example $\mathrm{C} 3$ or $\mathrm{C} 4$. When train we sing the pop songs, we teach the student to adjust their vocal cords thinner and thinner still, just with edge vibration. When we sing the highest pitch of countertenor, the edge vibration of the vocal cords, is the thinnest.

When we sing, the vocal cords are closed, but in consciousness, the two vocal cords are opened backwards. We should imagine that there's only one point in the two vocal cords that vibrates, over the pitch C3. Therefore, the throat can be fully opened and the sound is not jammed (Hudson, 1971).

Forth, the students should adjust the high position of the sound.

In the training of countertenors, we should pay attention to the adjustment of high position, while the high position adjustment of the countertenor technique can make the voice of popular singing more transparent, and more unified position from the lower pitch to the higher pitch.

In the head cavity, the high position is the reflection point of the intersection between the sound line of the vocal cords and of the hard palate. The reflex point is in the nasopharyngeal cavity while we train the students to sing the bass in popular songs. When students sing the higher pitch of popular songs, we hang the sound position in the nasopharyngeal cavity and the reflection point is backward. The higher the pitch, the more backward it is, especially when it comes to the height of the countertenor (For example: Figure 2). While adjusting the body's instruments and breath, we imagine that high position is like a plane landing on top of the pharynx.

\subsection{The Function Training of Vocal Cords}

Countertenor not only needs to strengthen the function of the pitch, needs to strengthen the function of the vocal state, but also needs to strengthen the vocal cords function. We use Peking Opera yelling voice method to practice the function of vocal cord. This kind of vocal cord function training method is used in the training of popular songs, whether it is the strength of the song or the high pitch, it is very necessary (Hansen, 1986). 


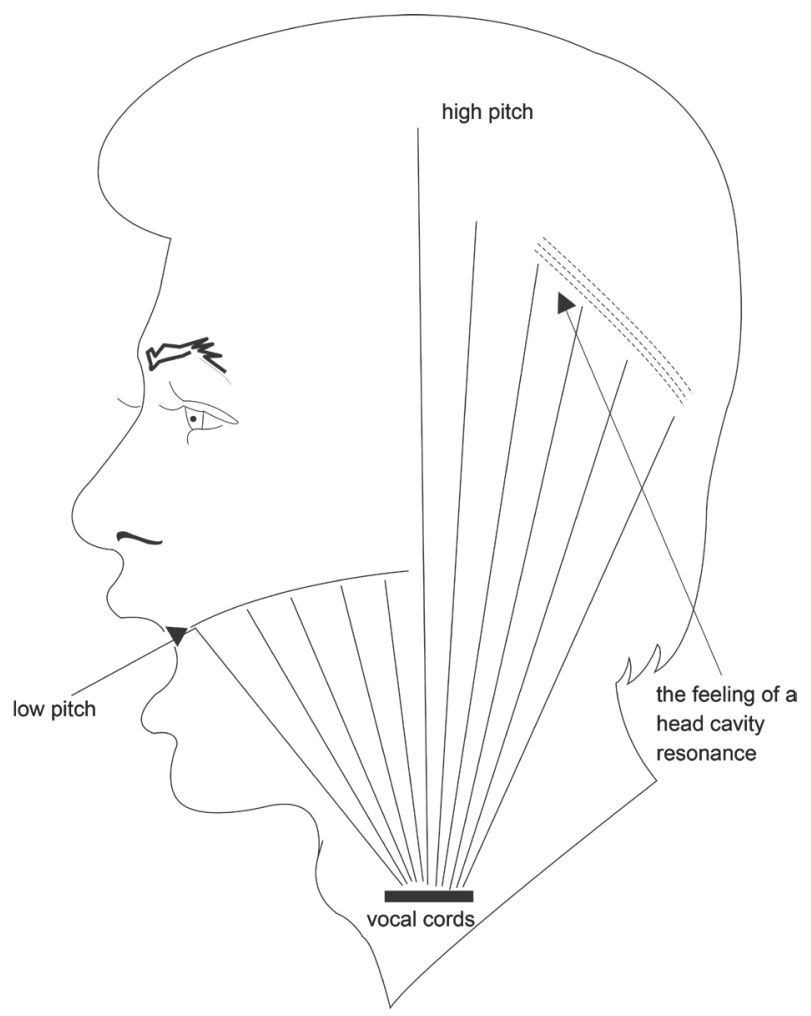

Figure 2. The resonance high position picture.

\section{Third Edition 1980}

Beijing Opera singers practice the sound method, by yelling the voice can exercise the various vocal parts, to phonate correctly each vowel. Yelling the voice is usually in the early morning, in the open area, shout out "wu", "yi", "ah" and so on, from lower pitch to higher pitch, and then from higher pitch to lower pitch, and repeated. After the sound is released, practice with the singing section.

Combined with the method of yelling the voice of Peking Opera, we phonate "mo" to establish the vocal body instrument, and then yell the " $\mathrm{A}$ " vowel under the protection of the vocal body instrument. We practice three minutes a day, from the fully vibration of the vocal cords to the local vibration, to the edge vibration, and then from the edge vibration, to the local vibration, to the full vibration of the pitch.

\subsection{The Lyrics Pronunciation Training}

When the falsetto tenor's body instrument is established and adjusted, the function training is enhanced every day, we use these skills in popular songs, which should be combined with the language of the lyrics. And each country's language is different, but all of them are made of consonants and vowels spelling. Exhale and obstruct when we pronounce consonants, and then, inhale and swallow when we pronounce vowels.

We start with the sound "mo" to build the vocal body instrument, and practice A, E, I, O, U to practice five vowels without affecting the sound state of the 
instrument, and practice consonants and vowel sounds spelling with the same method.

\section{Conclusion}

Vocal art is often the sublimation of vocal technique, especially for countertenor, which is the most technical part of vocal music. Singing is without borders, its beauty should be diversified. When we are in the training of singing popular songs, if the proper use of countertenor singing skills, not only can we get the tone of the countertenor, but we can also expand our vocal range. Therefore, we should master the countertenor skills through the four training steps, which are above-mentioned.

In addition to emotional expression, the performance of pop singers is often evaluated through the range of timbre, range, pitch and voice. This article discusses how to use countertenor technique in popular songs singing training skills, and it will also occupy more important positions in music market. Then in the future there will be more development for popular singing in the field of vocal music, I will analyze this kind of technique in some concrete vocal works.

\section{Conflicts of Interest}

The authors declare no conflicts of interest regarding the publication of this paper.

\section{References}

Burkholder, J. P. (2006). History of Western Music. New York, NY: W. W. Norton \& Company.

Hansen, P. S. (1986). An Introduction to Twentieth Century Music. Boston: Allyn and Bacon, Inc.

Hudson, R. (1971). The Ripresa the Ritornello and the Passacaglia. Journal of the American Musicological Society, 24, 364-394.

Stein, L. (1959). The Passacaglia in the Twentieth Century. Music and Letters, XL, $150-153$. 